\title{
Research on 3D Face Geometric Modeling Approach Based on Scattered-point Cloud
}

\author{
SHI LI-juan ${ }^{1.2}$, ZHAO Jian ${ }^{3}, \mathrm{CHE} \mathrm{Na}^{3}$ and AN ZHI-yong ${ }^{1} *$ \\ 1. College of Optoelectronic Engineering Changchun University of Science and \\ Technology, Changchun 130022 ,China \\ 2. College of Electronic Information and Engineering, Changchun University, \\ Changchun 130022, China \\ 3 College of Computer Science and Technology, Changchun \\ University, Changchun 130022, China) \\ *C47850117@qq.com
}

\begin{abstract}
With noise and plenty of redundant points, point cloud data obtained from non-contact scan method affects the reconstruction of geometric model. In view of the fact that denoising and simplification of original point cloud data serve as key points of geometric reconstruction, this paper, based on noise properties, puts forward a method to eliminate noise points at different scales through combination of chord height error and improved filter operator, and introduces an algorithm to carry out non-uniform simplification of point cloud based on bounding box and curvature fusion, through which feature points can be reserved in the process of eliminating redundant point cloud, and reduced points cloud data can thus be obtained. Finally, a geometrical face model is established through integration of multi-view point cloud in different angles and data registration. It can be demonstrated through experiments that this method can improve the arithmetic speed with the reconstruction integrity guaranteed and establish highly realistic $3 D$ face model with structural integrity and smooth surface.
\end{abstract}

Keywords: Point Cloud De-noising; Point Cloud Simplification; Space Partition; Curvature Estimation; Geometric Modeling

\section{Introduction}

In recent years, 3D face modeling is a research hotspot in the field of computer vision and computer graphics, and has been widely used in motion pictures \& television, manmachine interaction, computer game, communication and other areas ${ }^{[1]}$. Specific to rehabilitation of hearing-impaired children, this paper focuses on rehabilitation training for hearing-impaired child with 3D conversation avatar. To establish a 3D face model with a strong sense of reality, it's the precondition and basis for 3D conversation avatar to establish a real geometrical face. With the purpose of 3D face reconstruction, face data is obtained with a fast and efficient spatial digitizer; however, laser glitch, cavity and other problems come after multiple and disorderly point cloud data obtained from laser scanning. As a result, point cloud is mixed with some outliers and noise, and the reconstruction efficiency of feature extraction and facial surface is seriously affected ${ }^{[2]}$. Therefore, point cloud must be preprocessed before it is used to reconstruct 3D models. Domestic and foreign scholars have carried out plenty of researches on preprocessing of point cloud and raised many point cloud simplification methods. Traditional data simplification methods mainly consist of space division and curvature-based simplification method. For example, the former covers bounding box method ${ }^{[3]}$, uniform network method[4] and triangle-mesh method[5]. While the latter covers average distance 
method[6], curvature sampling method[7], angular deviation method[8], etc. There are certain limitations in both types of methods. Space division method is applicable to point cloud data with slight change in the characteristic curvature of flat curve only[9], while curvature-based simplification method has a lower computational efficiency even though geometrical characteristics can be reserved properly[10]. Therefore, how to acquire a higher simplification efficiency while reserving essential feature information of point cloud is the key technical issue of point cloud data preprocessing.

In addition, because of the complexity of human faces and familiarity of people with human faces, how to reconstruct an efficient and simplex 3D geometrical face model with processed point cloud data is also a difficulty.

Aiming at the aforementioned problems, the author firstly eliminates noise points through combination of chord height error algorithm and bilateral filter operator at different scales, simplifies non-uniform point cloud data with space partition and curvature, constructs the largest surrounding space, divides the space, searches adjacent areas with k-tree and establishes a micro-tangent surface, who then determines the normal vector, fits the curve and estimates the local curvature and sets the curvature threshold value, in which bounding box method is used for points whose curvature value is smaller than the threshold value. Moreover, curvature simplification method is used for those whose curvature value is larger than the threshold value. Besides, essential feature information of point cloud is reserved so as to improve the accuracy of surface features, which realizes a higher simplification efficiency while reserving the original surface features. Finally, a realistic 3D geometrical face model is obtained from the 3D face based on fundamental mesh deformation, and the compact and normative geometrical model outputted can be directly used to drive 3D model of hearing-impaired children rehabilitation systems.

\section{Space Partition of Point Cloud}

In this paper, original point cloud data is obtained with 3D face scanner of Beijing Bowei Hengxin and then such data is imported, among which there is no explicit geometry topology relation. In order to raise the search speed of area adjacent to scattered data points, the author carries out space partition to point cloud and divides point cloud through constructing the parting plane with bounding box method ${ }^{[11]}$, for which the partition process is shown below:

\subsection{Establishment of Bounding Box}

Lengths in three directions of the large bounding box are the differences between the maximum and the minimum. In order to place data points on the bounding box, error $\delta$ is given, and then all sides of the bounding box are migrated outside upon $\delta$ so as to obtain the original bounding space. With the longest axis in the original bounding space as the partition axis and the partition normal vector parallel to the longest axis, the partition space divides the point cloud space into two equal subspaces. Suppose $L_{x}$ is the projected length of 3D point cloud $\left(x_{i}, y_{i}, z_{i}\right)$ on axis $\mathrm{X}$, the plane which is parallel to the largest axis among $L_{x}, L_{y}$ and $L_{z}$ is divided, and kd-tree structure of 3D point cloud is established based on the above method, through which the point cloud space is divided into $\mathrm{n}$ subspaces and each has a reference point set $X_{1}^{n}\left(p_{i}\right)$ which is saved in the array.

\subsection{K-Neighborhood Calculation}

Space partition corresponds to a set of nodes, $p_{i}, p=\left\{p_{1}, p_{2}, \cdots p_{n}\right\} p_{i} \in R^{3}$. Search current space and inquire 26 adjacent cubes, and then determine $\mathrm{k}$ data points 
$\left\{\left(x_{t}, y_{t}, z_{t}\right)\right\}_{t=1}^{k}$ of $p_{i}$ in K-eighborhood, which is recorded as $N_{t=1}^{k}\left(p_{t}\right)$. When $k=24-32$, the curve has a higher precision. An excessively small value cannot reflect local geometrical features of point cloud data so that the estimation accuracy of vector and curvature will be lowered; while oversized adjacent data will increase the calculated amount of curvature $^{[12]}$. In this paper, the avatar model is reestablished, and the familiarity with the avatar requires a higher sense of reality, so $\mathrm{k}$ is supposed to be 32 .

\section{Point Cloud De-Noising}

\subsection{Removing Long-Distance Noise Points}

Specific to dense surface points of face and great change of characteristic position curvature, long-distance noise points are removed through chord height error algorithm ${ }^{[13]}$. The point cloud data set is defined $p_{i}$ as,and $p_{i+1}$ before and after the detected point $p_{i}$ are connected, and the distance $\lambda$ between the detected point and the connecting line is calculated. In addition, $\varepsilon$ is the given permissible deviation. If $\lambda\rangle \varepsilon$, $p_{i}$ is deemed as a noise point and should be removed.

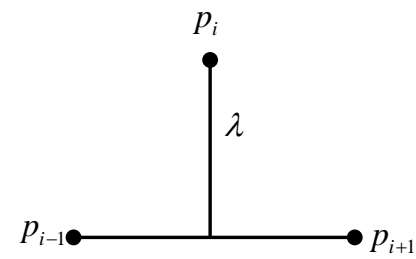

Figure 1. Chord Height Error Algorithm

\subsection{Removing Short-Distance Noise Points}

Short-distance noise in point cloud is mainly caused by small laser glitches in the scanning process. With similar features, such noise is high-frequency information to some extent, so feature information of point cloud cannot be lost in the de-noising process. On account of low robustness, common bilateral filtering algorithms depend on feature information of local neighborhoods, and multiple iterations will cause excessive smoothing of model. The author adopts the improved bilateral filter operator ${ }^{[14]}$ to enhance its robustness and characteristic preservation of such operator, and the improved bilateral filter operator is:

$$
d=\frac{\sum_{p_{i} \in p} W_{C}\left(\left\|p_{i}-p\right\|\right) W_{s}\left(\|\left\langle\vec{n}, p_{i}-p\right\rangle\right\rangle \|\left\langle\vec{n}, \vec{n}_{i}\right\rangle}{\sum_{p_{i} \in p} W_{C}\left(\left\|p_{i}-p\right\| W_{s}\left(\left\|\left\langle\vec{n}, \overrightarrow{n_{i}}\right\rangle\right\|\right)\right.}
$$

Where, $\vec{n}$ is the normal vector of point $p ; p_{i}$ is a point in $p,\left\langle\vec{n}, p_{i}-p\right\rangle$ is the dot product of two vectors; $W_{C}(x)$ is the Gaussian weighting function of spatial distance which controls the smooth degree. The larger $W_{C}(x)=e^{-x^{2} / 2 \sigma_{c}^{2}}, x=\left\|p_{i}-p\right\|$ and $W_{C}(x)$ are, the better the smooth effect is. $W_{s}(y)$ is the Gaussian kernel function of the normal angle 
of two points, for which the larger $y=\left\|\vec{n}-\overrightarrow{n_{i}}\right\| W_{s}(y)=e^{y^{2} / 2 \sigma_{s}^{2}}$, and $W_{s}(y)$ are, the stronger the feature preservation property is.

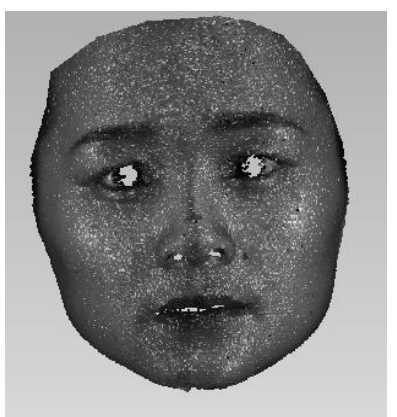

(a)original image

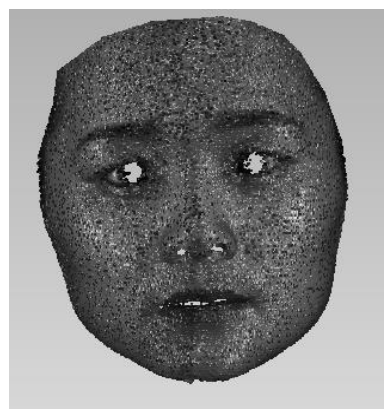

(b)Detected Noise Point Image

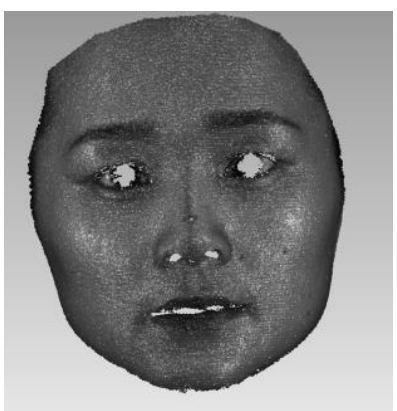

(c)Filter out Noise Point Image

Figure 2. Point Cloud Data after De-Noising

\section{Point Cloud Simplification}

\subsection{Vector Estimation}

The micro-tangent plane $T\left(u_{i}, v_{i}\right)$ of $p_{t}(t=1,2, \cdots k)$ is generated, then the quadratic sum of the distance from all points in the data point $p_{t}(t=1,2, \cdots k)$ to the plane is minimized through using equation

$$
\sum_{i=1}^{k}\left[p\left(x_{i}, y_{i}\right)-z_{i}\right]=\min \sum_{i=1}^{k}\left[p\left(x_{i}, y_{i}\right)-z_{i}\right]^{2}
$$

Generally, the plane form is

$$
p(x, y)=c_{00}+c_{10} x+c_{01} y+c_{20} x^{2}+c_{11} x y+c_{02} y^{2}
$$

Where, the projection of point set $\left\{\left(x_{i}, y_{i}, z_{i}\right)\right\}_{i=1}^{k}$ on the micro-tangent plane is $\left\{\left(x_{i}, y_{i}, z_{i}\right)\right\}_{i=1}^{k}$ If the projection $p^{\prime}$ of point $p$ is the coordinate origin, and the vector quantity of the point between $p^{\prime}$ and the farthest point is $u$ The normal vector of the microtangent plane is $w$, and $v$ is determined by $v=u \times w v$ is the normal vector of the plane, and thus the local coordi- nate system is established.

4.2 Surface fitting

The author adopts the local molded surface of point cloud with curve Bezier ${ }^{[15]}$ of order $n \times m$ and establishes a coordinate system. Suppose $q_{t} \in X$ and 4D coordinate is $q_{t}=\left(x_{t}, y_{t}, z_{t}, r_{t}\right), t=(0,1, \cdots, k)$, based on which curve Bezier can be expressed using equation:

$$
\begin{aligned}
& S(u, v)=\left[B_{0, n}(u), B_{1, n}(u), \cdots B_{m, n}(u)\right]\left[\begin{array}{cccc}
p_{00} & p_{01} & \cdots & p_{0 m} \\
p_{10} & p_{11} & \cdots & p_{1 m} \\
\cdots & \cdots & \cdots & \cdots \\
p_{n 0} & p_{n 1} & \cdots & p_{n m}
\end{array}\right]\left[\begin{array}{c}
B_{0, m}(v) \\
B_{1, m}(v) \\
\cdots \\
B_{n, m}(v)
\end{array}\right] \\
& \text { Suppose } \\
& A_{i, j}(u, v)=B_{i, n}(u) B_{j, m}(v)(i=0,1,2, \cdots n),(j=0,1,2, \cdots m),
\end{aligned}
$$

then, 
$S\left(u_{t}, v_{t}\right)=A_{i, j}(u, v) p_{i, j}=q(t)$

Where, $p_{i, j}$ is the control point of curve Bezier to be figured out.

When Formula (6) is expressed as

$$
A\left[\begin{array}{llll}
p_{1} & p_{2} & \cdots & p_{n}
\end{array}\right]^{T}=\left[\begin{array}{llll}
q_{0} & q_{1} & \cdots & q_{k}
\end{array}\right]^{T}
$$

then the control point of the method of least Surface is,

$\left[\begin{array}{llll}p_{1} & p_{2} & \cdots & p_{n}\end{array}\right]^{T}=A^{-1}\left[\begin{array}{llll}q_{0} & q_{1} & \cdots & q_{k}\end{array}\right]^{T}$

where,

$$
A^{-1}=V W^{-1} U^{-1}
$$

$U$ is the column orthogonal matrix of order $(k+1) \times[(m+1)(n+1)]$;

$W$ is the positive semidefinite diagonal matrix of order $[(m+1)(n+1)] \times[(m+1)(n+1)]$;

$V$ is the orthogonal matrix of $[(m+1)(n+1)] \times[(m+1)(n+1)]$

\subsection{Curvature Estimation in Adjacent Areas}

The normal of curve $S(u, v)$ at $p(x, y, z)$ is defined as $\vec{n}=\frac{N}{|N|}$, where, $N=S_{u} S_{v}$,

$S_{u}=\frac{\partial S(u, v)}{\partial u}, S_{u}=\frac{\partial S(u, v)}{\partial v}$.

Gaussian curvature

$$
K=\frac{\left(S_{u} S_{u} \cdot \vec{n}\right)\left(S_{v} S_{v} \cdot \vec{n}\right)-\left(S_{u} S_{v} \cdot \vec{n}\right)^{2}}{\left(S_{u} \cdot S_{u}\right) \cdot\left(S_{v} \cdot S_{v}\right)-\left(S_{u} \cdot S_{v}\right)^{2}}
$$

Average curvature

$$
\bar{K}=\sum_{i=1}^{n}\left|k_{i}\right|
$$

Curvature standard deviation

$$
\sigma=\sqrt{\sum_{i=0}^{n}(k-\vec{k})^{2} / /}
$$

The curvature threshold value is $\sigma$, for which the larger $\sigma$ is, the steeper the molded surface is. If $\sigma_{0}$ is smaller and points confined to $\sigma_{0}$ are deleted, then the smaller $\sigma$ is, the flatter the curve surface is. Through simplifying with bounding box algorithm and calculating

$$
d_{i}=\sqrt{\left(x_{i}-x\right)^{2}+\left(y_{i}-y\right)^{2}+\left(z_{i}-z\right)^{2}}=\min d_{i}
$$

with $o(x, y, z)$ as the center coordinate, the point is reserved and others in the bounding box are deleted.

\subsection{Point Cloud Simplification}

Flow of point cloud curvature simplification algorithm, As shown in Figure 3. 


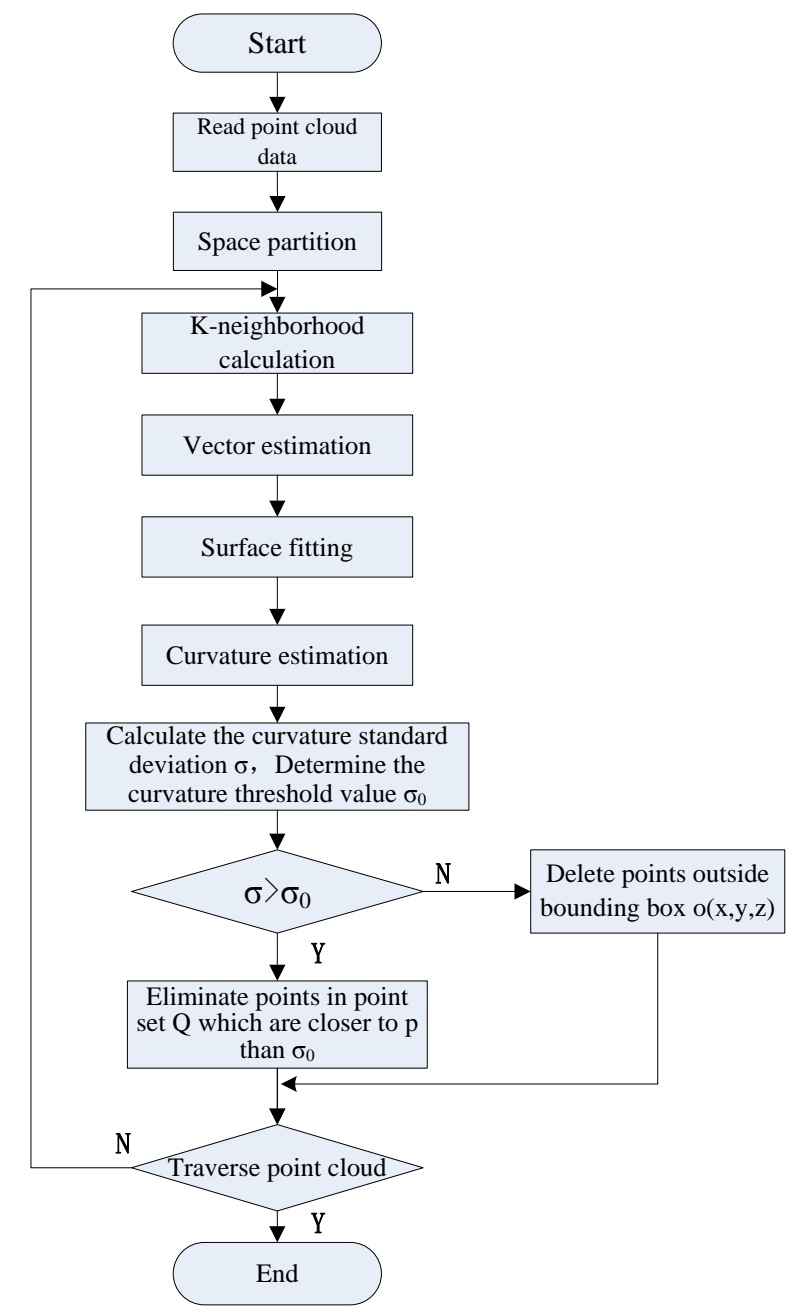

Figure 3. Flow of Curvature-based Point Cloud Simplification Algorithm

The Figure 4 (a)is the point cloud data reduction which is realized by the fusion of space and curvature.Point cloud data reduction using the fusion of space and curvature algorithm, The Figure 4 (b)is selecting a different threshold of different curvature of the two point cloud effect chart in the process of streamline.
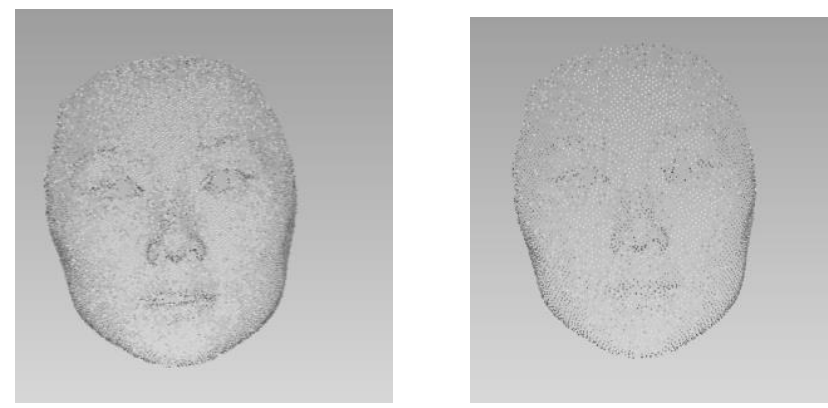

(a) Uniform Simplification Algorithm to Achieve the Point Cloud Data Effect Diagram 

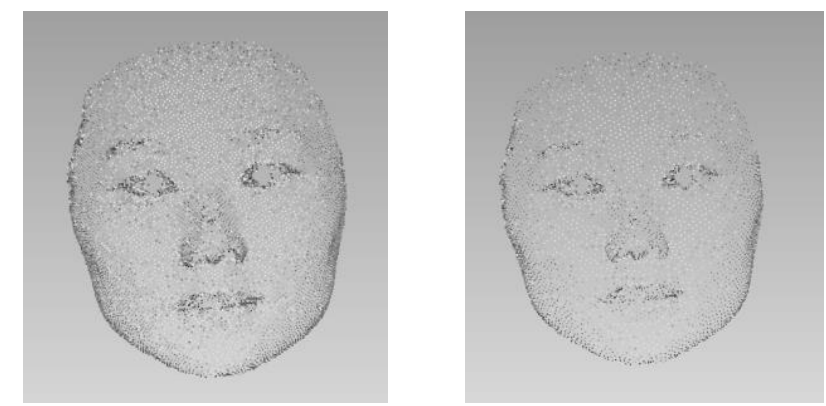

(b)Non uniform simplification algorithm of point cloud data effect diagram

\section{Figure 4. Effect Picture of Point Cloud Data Uniform and Non Uniform Simplification}

Through the Figure 4 (a)and (b)contrast can be seen with the streamline method than the uniform method of simplifying the curvature of the eyes, lips, nose and other regional retained more of the point cloud data, retained more clear contour feature, is conducive to the surface reconstruction.

\section{Modeling of Realistic 3D Geometrical Face Model}

Geometrical face model, which is established through integration and data registration of multi-view point cloud in different angles, is an unrealistic face with continuous curve surfaces and cannot be used for facial animation. Through a 3D face texture mapping method, real color and texture information are added to 3D face mesh and a realistic 3D geometrical face model can be established.
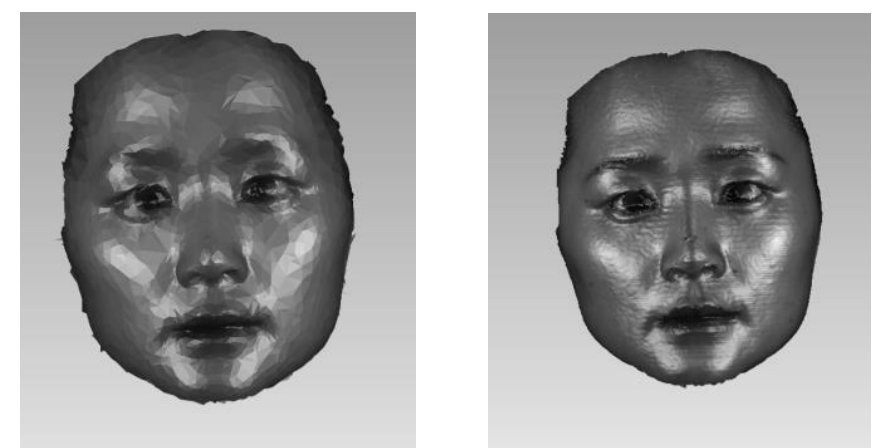

Figure 5. Colored and Textured 3D Geometrical Face Model

\section{Conclusion}

In this paper, 3D scanner is used to acquire 3D point cloud data, and de-noising and point cloud simplification are carried out specific to original point cloud data. Result of experiment demonstrates that 3D face model, which is established based on this method, features structural integrity and smoothing surface as well as strong sense of reality, and can thus be used in 3D conversation avatar of rehabilitation for hearing-impaired children.

\section{Acknowledgements}

This work was supported in part by NSFC under Grant No. 61502052; China Postdoctoral Science Foundation under Grant No. 2013M541298; The Fund of Ministry of Education of ChinaNo.Z2012062; Jilin Province Science and Technology Development Plan under Grant No. 20130522162JH; JiLin Province Education 
Development Plan under Grant Nos. 2016291,2015LY505L21. Changchun Administration of Science \&Technology Plan under Grant No.13GH03.

\section{References}

[1] Z. Shunlan, M. Jianwen and Z. Lulu, "Journal of Wuhan University of Technology", vol. 3, no. 38, (2014).

[2] B. Li, R. Schnabel and R. Klein, "Robust normal estimation for point cloud with sharp features", Computes Graphics, vol. 2, no. 34, (2010).

[3] J. Tao, C. J. Ting and F. Li, "Equipment Manufactring Technology", no. 3, (2010).

[4] K. H. Lee, H. Woo and T. Suk, "Point data reduction using 3D grids", The International Journal ofAdvanced Manufacturing Technology, vol. 3, no. 18, (2001).

[5] L. Yuzhen, "Research on Some Key Technoligies of 3D Point Cloud Data Processing", vol. 3, (2013), pp. 23-26.

[6] C. Moenning and N. A. Dodgson, "A New Point Cloud Simplification Algorithm”, Iasted Conference on Visualization, (2003), pp. 1027--1033.

[7] M. A. Zhen-Guo, "A point cloud simplification algorithm based on kd_tree and curvature sampling", Science of Surveying \& Mapping, vol. 35, no. 6, (2010).

[8] P. F. Lee and B. S. Jong, "Point-based simplification algorithm", Wseas Transactions on Computer Research, vol. 3, no. 1, (2008), pp. 61-66.

[9] K. K. Sareen, G. K. Knopf and R. Canas, "Contour-based 3d point data simplification for freeform surface reconstruction", International Journal of Shape Modeling, vol. 15, no. 2, (2011).

[10] K. K. Sareen, G. K. Knopf and R. Canas, "Contour-based 3D point cloud simplification for modeling freeform surfaces", Science and Technology for Humanity (TIC-STH), 2009 IEEE Toronto International Conference, (2009), pp. 381 - 386.

[11] H. Song and H. Y. Feng, "A progressive point cloud simplification algorithm with preserved sharp edge data", International Journal of Advanced Manufacturing Technology, vol. 45, no. 5-6, (2009), pp. 583592.

[12] H. Li, P. Xu and Y. Shen, "A self-adaption fast point cloud simplification algorithm based on normal eigenvalues", International Congress on Image and Signal Processing, IEEE, (2014), vol. 156, pp. 92937.

[13] Z. Chen and F. Da, S. O. Automation and S. University, "3d point cloud simplification algorithm based on fuzzy entropy iteration", Acta Optica Sinica, vol. 33, no. 8, (2013).

[14] L. Qingmeng, "Research on processing technology of point cloud from laser scanning system", vol. 3, (2013), pp. 23-26.

[15] S. Dianzhu, Z. Changzhi and F. Zhixian, "China Mechanical Engineering", vol. 23, (2009), pp. 28402843. 